\title{
NÍVEL DE ATIVIDADE FÍSICA EM ADOLESCENTES SAUDÁVEIS
}

\author{
PHYSICAL ACTIVITY IN HEALTHY ADOLESCENTS
}

NIVEL DE ACTIVIDAD FISICA EN ADOLESCENTES SANOS

Artigo Original

Original Article

Alexandre de Paiva Luciano ${ }^{1,2}$ (Médico)

Ciro João Bertoli ${ }^{3}$

(Médico)

Fernando Adami ${ }^{1}$

(Educador Físico)

Luiz Carlos de Abreu

(Fisioterapeuta)

1. Faculdade de Medicina do ABC (FMABC), Departamento de Saúde da Coletividade, Santo André, SP, Brasil.

2. Faculdade de Medicina de Taubaté, Departamento de Ortopedia, Grupo de Medicina Esportiva, Taubaté, SP, Brasil.

3. Faculdade de Medicina de Taubaté, Departamento de Pediatria e Adolescência, Taubaté, SP, Brasil.

\section{Correspondência:}

Instituto de Ortopedia Taubaté - IOT. Av. Nove de Julho 453. Centro Taubaté, SP, Brasil. 12020-900. paivaortopedia@gmail.com

\section{RESUMO}

Artículo Original

Introdução: A recomendação atual para prática de atividades físicas na infância e adolescência é de que todo jovem deveria envolver-se diariamente por 60 minutos ou mais em atividades físicas moderadas em cinco ou mais dias da semana, somando-se pelo menos 300 minutos de atividades físicas por semana. Poucos estudos sobre o nível de atividade física foram encontrados com amostras de crianças e adolescentes brasileiros. Objetivo: Esta pesquisa tem como objetivo a aplicação de um questionário para avaliar o nível de atividade física realizada e suas correlações com estágios da puberdade e índice de massa corporal (IMC) em adolescentes saudáveis matriculados na rede municipal de ensino da cidade de Taubaté, SP, Brasil. Métodos: Foi realizado um estudo transversal por meio de aplicação de um questionário (IPAQ) para avaliar o nível de atividade física e sua correlação com peso, altura e IMC de 202 adolescentes saudáveis. Resultados: O grupo de 15 a 17 anos, considerado pós-púbere nesta pesquisa, apresentou tempos significantemente maiores do que os demais grupos, quanto à resposta da questão $4 \mathrm{~b}(\mathrm{p}=0,002)$, que indaga sobre o tempo total gasto sentado durante um dia de final de semana. Conclusão: O grupo de 9 a 11 anos, considerado pré-púbere nesta pesquisa, apresentou números significantemente maiores do que os demais grupos quanto às atividades lúdicas moderadas.

Descritores: atividade física; exercício; adolescente.

\section{ABSTRACT}

Introduction: The current recommendation for physical activity in childhood and adolescence is that every young person should engage daily for 60 minutes or more in moderate physical activity on five or more days a week, totalizing at least 300 minutes of physical activity per week. Few studies on the level of physical activity were found in samples of Brazilian children and adolescents. Objective: This research aims to apply a questionnaire to assess the level of physical activity and its correlation with stages of puberty and body mass index (BMI) in healthy adolescents enrolled in municipal schools in the city of Taubaté, SP, Brazil. Methods: This is a cross-sectional study conducted through the application of a questionnaire (IPAQ) to assess the level of physical activity and its correlation with weight, height and BMI of 202 healthy adolescents. Results: The group of 15 to 17 years, considered post-pubertal in this study, showed significantly longer times than the other groups regarding the answer to the question $4 b(p=0.002)$, which inquires about the total time spent sitting during a day of the weekend. Conclusion: The group 9-11 years, considered pre-pubertal in this study, showed significantly longer times than the other groups with regard to moderate ludic activity.

Keywords: physical activity; exercise; adolescent.

\section{RESUMEN}

Introducción: La recomendación actual para la actividad física en la infancia y la adolescencia es que todos los jóvenes deben participar todos los días durante 60 minutos o más de actividad física moderada, cinco o más días de la semana, sumando por lo menos 300 minutos de actividad física semanal. Se encontraron pocos estudios sobre el nivel de actividad física en muestras de niños y adolescentes brasileños. Objetivo: Esta investigación tiene como objetivo aplicar un cuestionario para evaluar el nivel de actividad física realizada y su correlación con las etapas de la pubertad y el índice de masa corporal (IMC) en adolescentes sanos inscritos en las escuelas municipales en la ciudad de Taubaté, SP, Brasil. Métodos: Se realizó un estudio transversal mediante la aplicación de un cuestionario (IPAQ) para evaluar el nivel de actividad física y su correlación con el peso, la altura y el IMC de 202 adolescentes sanos. Resultados: El grupo de 15 a 17 años, considerado pospuberal en este estudio, tuvo tiempos significativamente más largos que los otros grupos, según la respuesta a la pregunta $4 b(p=0,002)$, que indaga sobre el total de tiempo dedicado a estar sentado durante un día del fin de semana. Conclusión: El grupo de 9 a 11 años, considerado prepuberal en este estudio, tuvo tiempos significativamente más largos que otros grupos con respecto a las actividades lúdicas moderadas.

Descriptores: actividad física; ejercicio; adolescente.

\section{INTRODUÇÃO}

No Brasil o estado nutricional das crianças, adolescentes e adultos têm se modificado nas últimas décadas passando da predominância de doenças infectocontagiosas, com repercussões nutricionais, para uma maior prevalência de doenças crônicas não transmissíveis ${ }^{1,2}$.
A redução no gasto energético (inatividade física) em crianças e adultos é o determinante mais importante do sobrepeso, e não é difícil verificar que as três principais alterações desta variável no estilo de vida têm ocorrido entre os jovens, ao longo de décadas recentes ${ }^{3}$. 
Atividade física regular apresenta relação inversa com peso corporal ${ }^{4}$ e com doenças crônicas não transmissíveis ${ }^{5,6}$, além de promover benefícios na aptidão física ${ }^{7,8}$, na maximização do pico de massa mineral óssea na adolescência e início da idade adulta ${ }^{9}$. A prática regular de atividade física auxilia na promoção da saúde e melhora da qualidade de vida de crianças e adolescentes, além de ser primordial para manutenção deste hábito na idade adulta.

A recomendação atual para prática de atividades físicas na infância e adolescência é de que todo jovem deveria envolver-se diariamente por 60 minutos ou mais de atividades físicas moderadas em cinco ou mais dias da semana ${ }^{10,11}$, somando-se pelo menos 300 minutos de atividades físicas por semana. Entretanto, estudos de intervenção realizados em grandes centros urbanos demonstram que mais de $50 \%$ das crianças e adolescentes não atingem as recomendações atuais de atividade física ${ }^{12-14}$.

Poucos estudos sobre o nível de atividade física foram encontrados com amostras de crianças e adolescentes brasileiros ${ }^{15-17}$.

Portanto, esta pesquisa tem como objetivo a aplicação de um questionário (IPAQ) ${ }^{18}$ para avaliar o nível de atividade física realizada e suas correlações com estágios da puberdade e índice de massa corpórea (IMC) em adolescentes saudáveis matriculados na rede municipal de ensino da cidade de Taubaté, SP, Brasil.

\section{CASUÍSTICA E MÉTODOS}

Foi realizado um estudo transversal através da aplicação de um questionário (IPAQ) ${ }^{18}$ (anexo 1) para avaliar o nível de atividade física e sua correlação com peso, altura e IMC de 202 adolescentes saudáveis praticantes de atividade física matriculados na rede municipal de ensino da cidade de Taubaté, SP, Brasil. Foi proposto um grupo, optou-se por realizar o estudo na maior e mais populosa das escolas da rede municipal da cidade de Taubaté, SP, Brasil. Optou-se pela divisão por grupo etário de 9 - 11 anos (pré púberes); 12 - 14 (púberes) e 15 - 17 anos (pós - púberes). O termo de consentimento livre esclarecido (TCLE) foi distribuído e assinado pelos responsáveis. Esta pesquisa foi avaliada e aprovada pelo Comitê de Ética em Pesquisa da Universidade de Taubaté CEP/Unitau no 102/11 sob o número 273/11.

Para verificação do Índice de Massa Corporal (IMC = peso $(\mathrm{kg}) /$ $\operatorname{altura}^{2}(\mathrm{~m})$, foi realizada medida de peso e estatura. Os adolescentes foram pesados sem sapatos, e com o mínimo de roupa possível, em balança eletrônica. Para a medida de estatura, os adolescentes encostaram-se na parede, posicionando a cabeça de acordo com o plano de Frankfurt. Foi utilizado um estadiômetro fixado à parede. As medidas foram realizadas pelo mesmo avaliador.

Os Critérios de inclusão foram adolescentes saudáveis do sexo masculino entre 12 e 17 anos que praticam atividade física regularmente.

Os Critérios de exclusão foram adolescentes do sexo feminino, devido a desordens hormonais e ciclos menstruais que nestas faixas etárias estudadas, podem alterar a fidedignidade dos achados. Adolescentes entre 12 e 17 anos, com doenças crônicas degenerativas, má formações congênitas, patologias neurológicas, com doenças cardiorrespiratórias e/ou ortopédicas.

As variáveis foram resumidas por grupo de estudo pelas estatísticas descritivas pertinentes: média, desvio padrão (dp), mediana, valores mínimo e máximo para as variáveis numéricas e frequências absoluta e relativa (\%) para as variáveis categóricas.

Foi aplicado o teste não paramétrico de Kruskal-Wallis para amostras independentes na comparação entre os grupos quanto às respostas do questionário.

Em testes com resultado estatisticamente significante, foi aplicado o teste de comparações múltiplas de Dunn, para localizar os grupos diferentes.

Todas as análises estatísticas foram executadas pelo software estatístico SPSS for Windows versão 18.0.

Foi adotado o nível de significância de 0,05 ( $a=5 \%$ ) e níveis descritivos ( $p$-valores) inferiores a esse valor foram considerados significantes.

\section{RESULTADOS}

A Tabela 1 mostra a descrição das medidas antropométricas segundo o grupo etário dos adolescentes apontando o grupo etário de 9 - 11 anos com 30,2\% de sobrepeso e $28,6 \%$ de obesos.

Na comparação entre os grupos etários quanto às médias dos números de dias e dos tempos respondidos no questionário foram encontrados os seguintes resultados (Tabela 2).

Foi encontrada diferença estatisticamente significante entre os grupos etários quanto ao número de dias respondido na questão 1a ( $p<0,001$ ), em que o grupo de 9 a 11 anos apresentou números de dias significantemente menores do que os demais grupos. Os grupos de 12 a 14 anos e 15 a 17 anos não se diferenciaram de forma significante.

Encontramos diferença estatisticamente significante entre os grupos etários quanto ao tempo respondido na questão $1 b(p=0,014)$, em que o grupo de 9 a 11 anos apresentou tempos significantemente menores do que os do grupo de 12 a 14 anos. O grupo de 15 a 17 anos não se diferenciou dos demais grupos de forma significante.

Observamos diferença estatisticamente significante entre os grupos etários quanto ao tempo respondido na questão $2 b(p=0,005)$, em que o grupo de 9 a 11 anos apresentou tempos significantemente maiores do que os demais grupos. Os grupos de 12 a 14 anos e 15 a 17 anos não se diferenciaram de forma significante.

Tabela 1. Medidas descritivas (média (dp), mediana, mínimo / máximo) das medidas antropométricas segundo o grupo etário dos adolescentes.

\begin{tabular}{c|c|c|c}
\hline \multirow{2}{*}{ Dados } & \multicolumn{3}{|c}{ Grupo etário } \\
\cline { 2 - 4 } & $\mathbf{9}$ a 11 anos & $\mathbf{1 2}$ a 14 anos & $\mathbf{1 5}$ a 17 anos \\
\cline { 2 - 4 } & $\mathbf{n = 6 3}$ & $\mathbf{n}=\mathbf{7 5}$ & $\mathbf{n = 6 4}$ \\
\hline \multirow{3}{*}{ Peso $(\mathrm{kg})$} & $44,0(12,0)$ & $55,6(13,3)$ & $66,6(13,5)$ \\
\cline { 2 - 4 } & 42,9 & 53,6 & 67,0 \\
\cline { 2 - 4 } & $28,0 / 72,2$ & $35,0 / 110,0$ & $40,0 / 91,0$ \\
\hline \multirow{3}{*}{ Altura $(\mathrm{m})$} & $1,43(0,11)$ & $1,63(0,08)$ & $1,68(0,06)$ \\
\cline { 2 - 4 } & 1,40 & 1,65 & 1,69 \\
\cline { 2 - 4 } & $1,28 / 1,64$ & $1,40 / 1,80$ & $1,50 / 1,79$ \\
\hline \multirow{3}{*}{ IMC $\left(\mathrm{kg} / \mathrm{m}^{2}\right)$} & $21,1(4,0)$ & $20,8(3,9)$ & $23,5(4,2)$ \\
\cline { 2 - 4 } & 20,0 & 19,8 & $14,0 / 31,5$ \\
\cline { 2 - 4 } & $14,9 / 29,7$ & $14,6 / 34,0$ & $13(20,3 \%)$ \\
\hline Classificação do IMC & & & $26(40,6 \%)$ \\
\hline Baixo peso & $5(7,9 \%)$ & $28(37,3 \%)$ & $8(12,5 \%)$ \\
\hline Normal & $21(33,3 \%)$ & $22(29,3 \%)$ & \\
\hline Sobrepeso & $19(30,2 \%)$ & $20(26,7 \%)$ &
\end{tabular}

Tabela 2. Medidas descritivas (média (dp), mediana, mínimo / máximo) das respostas do questionário segundo o grupo etário dos adolescentes.

\begin{tabular}{|c|c|c|c|c|}
\hline \multirow{3}{*}{ Questão } & \multicolumn{3}{|c|}{ Grupo etário } & \multirow{3}{*}{$p$-valor } \\
\hline & 9 a 11 anos & 12 a 14 anos & 15 a 17 anos & \\
\hline & $n=63$ & $n=75$ & $n=64$ & \\
\hline \multirow{3}{*}{$1 a$} & $2,25(2,09)$ & $3,84(2,19)$ & $3,97(2,45)$ & \multirow{3}{*}{$<0,001^{*}$} \\
\hline & 2,00 & 5,00 & 5,00 & \\
\hline & $0 / 7$ & $0 / 7$ & $0 / 7$ & \\
\hline \multirow{3}{*}{$1 b$} & $0: 35(041)$ & $0: 54(0: 45)$ & $0: 47(1: 01)$ & \multirow{3}{*}{0,014 * } \\
\hline & $0: 30$ & $0: 50$ & $0: 30$ & \\
\hline & $0: 00 / 4: 00$ & $0: 00 / 3: 00$ & 0:00 / 6:00 & \\
\hline \multirow{3}{*}{$2 a$} & $2,76(1,56)$ & $2,99(1,90)$ & $3,25(2,42)$ & \multirow{3}{*}{0,636} \\
\hline & 2,00 & 3,00 & 3,00 & \\
\hline & $0 / 6$ & $0 / 7$ & $0 / 7$ & \\
\hline \multirow{3}{*}{$2 b$} & $1: 30(1: 04)$ & 1:04 (0:47) & $0: 59(1: 07)$ & \multirow{3}{*}{$0,005^{*}$} \\
\hline & $1: 00$ & $1: 00$ & $1: 00$ & \\
\hline & $0: 00 / 4: 00$ & $0: 00 / 4: 00$ & $0: 00 / 6: 00$ & \\
\hline \multirow{3}{*}{$3 a$} & $1,35(1,61)$ & $2,37(1,93)$ & $2,23(2,15)$ & \multirow{3}{*}{0,002 * } \\
\hline & 1,00 & 2,00 & 2,00 & \\
\hline & $0 / 5$ & $0 / 7$ & $0 / 7$ & \\
\hline \multirow{3}{*}{$3 b$} & $0: 52(1: 04)$ & $1: 06(1: 06)$ & $0: 59(1: 01)$ & \multirow{3}{*}{0,187} \\
\hline & $0: 40$ & $1: 00$ & $1: 00$ & \\
\hline & $0: 00 / 4: 00$ & 0:00 / 6:30 & 0:00/ 5:30 & \\
\hline \multirow{3}{*}{$4 a$} & $6: 41(3: 00)$ & $6: 52(2: 34)$ & $7: 38(3: 29)$ & \multirow{3}{*}{0,329} \\
\hline & $6: 00$ & 7:00 & $8: 00$ & \\
\hline & $1: 30 / 15: 00$ & $0: 35 / 12: 00$ & 3:00 / 20:00 & \\
\hline \multirow{3}{*}{$4 b$} & $6: 15(3: 44)$ & $7: 38(3: 53)$ & $9: 52(5: 37)$ & \multirow{3}{*}{$0,002^{*}$} \\
\hline & $6: 00$ & $8: 00$ & $8: 00$ & \\
\hline & 1:00/15:00 & $2: 00 / 20: 00$ & $2: 00 / 20: 00$ & \\
\hline
\end{tabular}


Foi encontrada diferença estatisticamente significante entre os grupos etários quanto ao número de dias respondido na questão 3a ( $p=0,002)$, em que o grupo de 9 a 11 anos apresentou números de dias significantemente menores do que os demais grupos. Os grupos de 12 a 14 anos e 15 a 17 anos não se diferenciaram de forma significante.

Encontramos ainda diferença estatisticamente significante entre os grupos etários quanto ao tempo respondido na questão $4 b(p=0,002)$, em que o grupo de 15 a 17 anos apresentou tempo significantemente maior que os demais grupos. Os grupos de 9 a 11 anos e 12 a 14 anos não se diferenciaram de forma significante.

Não foi encontrada associação estatisticamente significante entre a classificação do nível de atividade física IPAQ (Tabela 3) e a classificação do IMC (Tabela 4) dos adolescentes avaliados ( $p=0,0667$ ).

\section{DISCUSSÃO}

Autores ${ }^{19,20}$ citam as evidências fisiológicas dos benefícios à saúde pela atividade física, especialmente quanto a doenças cardiovasculares, têm sua origem durante a infância e adolescência. Percebe-se, diante disso, a importância da relação entre a atividade física e estado de saúde dos adolescentes para o futuro.

Segundo Heath et al..$^{21}$, a participação de indivíduos em atividade física é reconhecida como um componente do estilo de vida saudável. Entre adolescentes, a participação em programas de atividade física se apresenta como um agente de prevenção de distúrbios físicos e orgânicos.

A atividade física torna-se, portanto, um importante determinante nas características físicas do adolescente.

Nesta pesquisa, optou-se por aplicação de um questionário para avaliar o nível de atividade física realizada e suas correlações, sabemos que a medida precisa da atividade física é considerada um desafio para os pesquisadores ${ }^{22}$. Diversos métodos são citados na literatura, entre os mais comuns estão, água duplamente marcada, calorimetria, frequência cardíaca, pedômetros, acelerômetros e questionários.

A utilização dos questionários, têm se mostrado até o momento, como o único método prático de se avaliar a atividade física capaz de alcançar um grande número de crianças e adolescentes, com aceitável acurácia ${ }^{23}$. A utilização de questionários, além de avaliar a situação atual da atividade física, possibilita estimular o aumento da atividade, a intervenção e orientação nas modificações dos hábitos de vida dos jovens, que podem ser avaliados periodicamente por estes práticos métodos ${ }^{24}$. Algumas limitações ao uso dos questionários estão relacionadas à idade da criança. Porém, estas questões podem ser solucionadas com a ajuda dos pais e participação dos professores para responder algumas questões.

E esta pesquisa nos chamou à atenção para a diferença estatis-

Tabela 3. Distribuição da Classificação do Nível de Atividade Física IPAQ segundo o grupo etário dos adolescentes.

\begin{tabular}{c|c|c|c}
\hline \multirow{2}{*}{ Dados } & \multicolumn{3}{|c}{ Grupo etário } \\
\cline { 2 - 4 } & $\mathbf{9}$ a 11 anos & $\mathbf{1 2}$ a 14 anos & $\mathbf{1 5}$ a 17 anos \\
\cline { 2 - 4 } & $\mathbf{n = 6 3}$ & $\mathbf{n = 7 5}$ & $\mathbf{n = 6 4}$ \\
\hline Classificação IPAQ & & & $22(34,4 \%)$ \\
\hline Muito ativo & $10(15,9 \%)$ & $21(28,0 \%)$ & $28(43,8 \%)$ \\
\hline Ativo & $25(39,7 \%)$ & $46(61,3 \%)$ & $5(7,8 \%)$ \\
\hline Irregularmente ativo A & $17(27,0 \%)$ & $5(6,7 \%)$ & $7(10,9 \%)$ \\
\hline Irregularmente ativo B & $9(14,3 \%)$ & $2(2,7 \%)$ & $2(3,1 \%)$ \\
\hline Sedentário & $2(3,2 \%)$ & $1(1,3 \%)$ &
\end{tabular}

ticamente significante entre os grupos etários quanto as questão 1a ( $p<0,001), 1 b(p=0,014)$, em que o grupo de 9 a 11 anos, considerados pré-púberes nesta pesquisa, apresentou números de dias significantemente menores do que os demais grupos e a diferença estatisticamente significante entre os grupos etários quanto ao tempo respondido na questão 2b ( $p=0,005)$, em que, o grupo de 9 a 11 anos, apresentou tempos significantemente maiores do que os demais grupos. Estes resultados mostram a diferença do ritmo de crescimento em adolescentes em desenvolvimento na mesma idade e a época em que esse aspecto se torna mais nítido na puberdade. Para melhor avaliação do desenvolvimento dos caracteres sexuais secundários, podemos utilizar os critérios divulgados por Tanner JM (1962)25.

Encontramos diferença estatisticamente significante entre os grupos etários quanto ao tempo respondido na questão $4 b(p=0,002)$, que indaga sobre o tempo no total gasto sentado durante em um dia de final de semana. Em que o grupo de 15 a 17 anos, apresentou tempos significantemente maiores do que os demais grupos. Estes dados corroboram com a pesquisa de Dietz ${ }^{26}$ mostrando que a obesidade em adolescentes resulta do desequilíbrio entre atividade reduzida e excesso de consumo de alimentos densamente calóricos, tendo mostrado que o número de horas que um adolescente passa assistindo TV é um importante fator associado à obesidade. Autores também mostraram que grande número de horas sentado ou assistindo televisão está relacionado com os baixos níveis de atividade física, e pode ser associado ao acúmulo de gordura corporal em adolescentes ${ }^{27,28}$. A disponibilidade de tecnologia, o aumento da insegurança e a progressiva redução dos espaços livres nos centros urbanos (onde vive a maior parte das crianças brasileiras) reduzem as oportunidades de lazer e de uma vida fisicamente ativa, favorecendo atividades sedentárias, tais como: assistir televisão, jogar video-games e utilizar computadores, contribuindo para estes achados científicos.

A Sociedade Brasileira de Medicina do Esporte ${ }^{29}$ publicou o Posicionamento Oficial, mostrando que em crianças e adolescentes, um maior nível de atividade física contribui para melhorar o perfil lipídico e metabólico e reduzir a prevalência de obesidade. Ainda, é mais provável que uma criança fisicamente ativa se torne um adulto também ativo. Em consequência, do ponto de vista de saúde pública e medicina preventiva, promover a atividade física na infância e na adolescência, significa estabelecer uma base sólida para a redução da prevalência do sedentarismo na idade adulta, contribuindo desta forma para uma melhor qualidade de vida na idade adulta.

\section{CONCLUSÃO}

Concluímos que grupo de 15 a 17 anos, considerados pós-púberes nesta pesquisa, apresentou tempos significantemente maiores do que os demais grupos, quanto ao tempo respondido na questão $4 \mathrm{~b}$ $(p=0,002)$, que indaga sobre o tempo no total gasto sentado durante em um dia de final de semana.

Já o grupo de 9 a 11 anos, considerados pré-púberes nesta pesquisa, apresentou números significantemente maiores do que os demais grupos quanto as atividades moderadas de forma lúdica.

Todos os autores declararam não haver qualquer potencial conflito de interesses referente a este artigo.

Tabela 4. Associação entre a Classificação do Nível de Atividade Física IPAQ e a Classificação do IMC dos adolescentes.

\begin{tabular}{|c|c|c|c|c|c|c|}
\hline & \multicolumn{5}{|c|}{ Classificação IPAQ } & \multirow{2}{*}{ Total } \\
\hline & Muito ativo & Ativo & Irregular/e ativo $\mathrm{A}$ & Irregular/e ativo B & Sedentário & \\
\hline & $n=53$ & $\mathrm{n}=99$ & $\mathrm{n}=27$ & $n=18$ & $n=5$ & $n=202$ \\
\hline \multicolumn{7}{|c|}{ Classificação IMC } \\
\hline Baixo peso & $15(28,3 \%)$ & $26(26,3 \%)$ & $1(3,7 \%)$ & $3(16,7 \%)$ & $1(20,0 \%)$ & $46(22,8 \%)$ \\
\hline Normal & $20(37,7 \%)$ & $33(33,3 \%)$ & $10(37,0 \%)$ & $4(22,2 \%)$ & $2(40,0 \%)$ & $69(34,2 \%)$ \\
\hline Obeso & $4(7,5 \%)$ & $13(13,1 \%)$ & $9(33,3 \%)$ & $5(27,8 \%)$ & $0(0 \%)$ & $31(15,3 \%)$ \\
\hline Sobrepeso & $14(26,4 \%)$ & $27(27,3 \%)$ & $7(25,9 \%)$ & $6(33,3 \%)$ & $2(40,0 \%)$ & $56(27,7 \%)$ \\
\hline p-valor & \multicolumn{5}{|c|}{0,0667} & \\
\hline
\end{tabular}


CONTRIBUIÇÕES DOS AUTORES: Cada autor contribuiu individual e significativamente para o desenvolvimento do manuscrito. FA (0000-0003-1918-3364)* e LCA (0000-0002-7618-2109)* foram os principais contribuintes na redação e revisão do manuscrito. APL (0000-0001-9753-4727)* e CJB (0000-0002-5666-5189)* realizaram a distribuição dos questionários, acompanharam os adolescentes e reuniram medidas antropométricas, bioestatística e pesquisa bibliográfica. Por fim, APL, CJB, FA e LCA contribuíram com o conceito intelectual do estudo. *Número ORCID (Open Researcher and Contributor ID).

\section{REFERÊNCIAS}

1. Hatano S. Changing CHD mortality and its causes in Japan during 1955-1985. Int J Epidemiol. 1989;18(3 Suppl 1):S149-58.

2. Valiente S, Abala C, Avila B, Monckeberg F. Patologia Nutricional en America Latina y El Caribe. Arch Latinoam Nutr. 1988;38(3):445-65.

3. Seidell JC. A atual epidemia de obesidade. In: Bouchard C, editor. Atividade física e obesidade. Tradução de Dulce Marino. Barueri, SP: Editora Manole; 2002. p. 23-33.

4. Fernandez AC, Mello MT, Tufik S, Castro PM, Fisberg M. Influência do treinamento aeróbio e anaeróbio na massa de gordura corporal de adolescentes obesos. Rev Bras Med Esporte. 2004;10(3):159-64.

5. Ewart CK, Young DR, Hagberg JM. Effects of school-based aerobic exercise on blood pressure in adolescent girls at risk for hypertension. Am J Public Health. 1998;88(6):949-51.

6. Haines $L$, Wan $K C$, Lynn $R$, Barrett TG, Shield JP. Rising incidence of type 2 diabetes in children in the U.K. Diabetes Care. 2007;30(5):1097-101

7. Cooper AR, Wedderkopp N, Wang H, Andersen LB, Froberg K, Page AS. Active travel to school and cardiovascular fitness in Danish children and adolescents. Med Sci Sports Exerc. 2006;38(10):1724-31

8. Baquet $G$, Berthoin $S$, Gerbeaux M, Van Praagh E. High-intensity aerobic training during a 10 week one-hour physical education cycle: effects on physical fitness of adolescents aged 11 to 16 . Int J Sports Med. 2001;22(4):295-300

9. Ortega FB, Ruiz JR, Castillo MJ, Sjöström M. Physical fitness in childhood and adolescence: a powerful marker of health. Int J Obes (Lond). 2008;32(1):1-11.

10. Strong WB, Malina RM, Blimkie CJ, Daniels SR, Dishman RK, Gutin B, et al. Evidence based physical activity for school-age youth. J Pediatr. 2005;146(6):732-7.

11. Pate RR, Freedson PS, Sallis JF, Taylor WC, Sirard J, Trost SG, et al. Compliance with physical activity guidelines: prevalence in a population of children and youth. Ann Epidemiol. 2002;12(5):303-8.

12. Ceschini FL, Florindo AA, d'Aquino Benício MH. Nível de atividade física em adolescentes de uma região de elevado índice de vulnerabilidade juvenil. Rev Bras Cienc Mov. 2007;15(4):67-78.

13. Romero A, Slater B, Florindo AA, Latorre MRD, Cesar C, Silva MV. Determinantes do índice de massa corporal em adolescentes de escolas públicas de Piracicaba, São Paulo. Ciênc Saúde Colet. 2008; 15(1):142-9.

14. Reis RS, Hino AA, Florindo AA, Añez CR, Domingues MR. Association between physical activity in parks and perceived environment: a study with adolescents. J Phys Act Health. 2009;6(4):503-9.
15. Maitino EM. Aspectos de risco coronariano em casuística de crianças de escola pública de primeiro grau em Bauru, SP. Rev Bras Ativ Fís Saúde. 1997;2(1):37-52.

16. Andrade D, Araújo T, Matsudo SM, Matsudo VK, Andrade E, Rocha A, et al. Physical activity patterns in female Teenagers from different socioeconomic regions. In: Casagrande G, Viviani F, editors. Physical activity and health: physiological, behavioral and epidemiological aspects. Padova: Unipress; 1998. p. 115-22.

17. Matsudo VK, Matsudo SM, Andrade DR, Rocha A, Andrade E, Andrade R. Level of physical activity in boys and girls from Low socio-economic region. In: Casagrande G, Viviani F, editors. Physical activity and health: physiological, behavioral and epidemiological aspects. Padova: Unipress; 1998. p. 139-45.

18. Matsudo S, Araújo T, Marsudo V, Andrade D, Andrade E, Oliveira LC, et al. Questinário internacional de atividade física (IPAQ): estudo de validade e reprodutibilidade no Brasil. Rev Bras Ativ Fis Saúde. 2001;6(2):5-18.

19. Livingstone MB. Energy expenditure and physical activity in relation to fitness in children. Proc Nutr Soc. 1994;53(1):207-21.

20. Shephard RJ. Custos e benefícios dos exercícios físicos na criança. Rev Bras Ativ Fís Saúde. 1995;1 (1):66-84

21. Heath GW, Pate RR, Pratt M. Measuring physical activity among adolescents. Public Health Rep. 1993;108(Suppl 1):42-6.

22. Melby CL, Ho RC, Hill JO. Avaliação do gasto energético humano. In: Bouchard C, editor. Atividade física e obesidade. Tradução de Dulce Marino. Barueri, SP: Manole; 2002. p.117-50.

23. Booth ML, Okely AD, Chey T, Bauman A. The reliability and validity of the physical activity questions in the WHO health behaviour in schoolchildren (HBSC) survey: a population study. Br J Sports Med. 2001;35(4):263-7.

24. Shephard RJ. Limits to the measurement of habitual physical activity by questionnaires. Br J Sports Med. 2003;37(3):197-206

25. Tanner JM. Growth at adolescence. Oxford: Blackwell Scientific; 1962.

26. Dietz WH. Critical periods in childhood for the development of obesity. Am J Clin Nutr. 1994;59(5):955-9.

27. Silva RCR, Malina RM. Nível de atividade física em adolescentes do município de Niterói, Rio de Janeiro, Brasil. Cad Saúde Pública, Rio de Janeiro. 2000;16(4):1091-7.

28. Pinho RA. Nível habitual de atividade física e hábitos alimentares de adolescentes durante período de férias escolares [dissertação]. Santa Florianópolis: Universidade Federal de Santa Catarina; 1999.

29. Lazzoli JK, Nóbrega ACL, Carvalho T, Oliveira MAB, Teixeira JAC, Leitão MB, et al. Atividade física e saúde na infância e adolescência. Rev Bras Med Esporte. 1998;4(4):107-9.

Anexo I. Questionário internacional de atividade física - versão curta.

Nome:

Data:

/ 1 1 1

Peso:

Kg Altura:

m IMC:

Sexo: $M()$

Nós estamos interessados em saber que tipos de atividade física as pessoas fazem como parte do seu dia a dia.

As perguntas estão relacionadas ao tempo que você gasta fazendo atividade física na ÚLTIMA semana.

As perguntas incluem as atividades que você faz na escola ou no trabalho, para ir de um lugar a outro, por lazer, por esporte, por exercício ou como parte das suas

atividades em casa ou no jardim.

Suas respostas são MUITO importantes. Por favor, responda cada questão mesmo que considere que não seja ativo. Obrigado pela sua participação!

Para responder as questões lembre que:

- Atividades físicas VIGOROSAS são aquelas que precisam de um grande esforço físico e que fazem respirar MUITO mais forte que o normal.

- Atividades físicas MODERADAS são aquelas que precisam de algum esforço físico e que fazem respirar UM POUCO mais forte que o normal.

Para responder as perguntas pense somente nas atividades que você realiza por pelo menos 10 minutos contínuos de cada vez.

1a. Em quantos dias da última semana você CAMINHOU por pelo menos 10 minutos contínuos em casa ou no trabalho, como forma de transporte para ir de um lugar para outro, por lazer, por prazer ou como forma de exercício?

Dias__ por semana ( ) Nenhum.

1b. Nos dias em que você caminhou por pelo menos 10 minutos contínuos quanto tempo no total você gastou caminhando por dia?

Horas: minutos:

2a. Em quantos dias da última semana, você realizou atividades MODERADAS por pelo menos 10 minutos contínuos, como por exemplo pedalar leve na bicicleta, nadar, dançar, fazer ginástica aeróbica leve, jogar vôlei recreativo, carregar pesos leves, fazer serviços domésticos na casa, no quintal ou no jardim como varrer, aspirar, cuidar do jardim, ou qualquer atividade que fez aumentar moderadamente sua respiração ou batimentos do coração (POR FAVOR NÃO INCLUA CAMINHADA)

Dias por semana ( ) Nenhum.

2b Nos dias em que você fez essas atividades moderadas por pelo menos 10 minutos contínuos, quanto tempo no total você gastou fazendo essas atividades por dia? Horas: minutos:

3a. Em quantos dias da última semana, você realizou atividades VIGOROSAS por pelo menos 10 minutos contínuos, como por exemplo, correr, fazer ginástica aeróbica, jogar futebol, pedalar rápido na bicicleta, jogar basquete, fazer serviços domésticos pesados em casa, no quintal ou cavoucar no jardim, carregar pesos elevados ou qualquer atividade que fez aumentar MUITO sua respiração ou batimentos do coração.

Dias __ por semana ( ) Nenhum.

3b. Nos dias em que você fez essas atividades vigorosas por pelo menos 10 minutos contínuos quanto tempo no total você gastou fazendo essas atividades por dia? Horas: minutos:

Estas últimas questões são sobre o tempo que você permanece sentado todo dia, no trabalho, na escola ou faculdade, em casa e durante seu tempo livre. Isto inclui o tempo sentado estudando, sentado enquanto descansa, fazendo lição de casa visitando um amigo, lendo, sentado ou deitado assistindo TV. Não inclua o tempo gasto sentando durante o transporte em ônibus, trem, metrô ou carro.

4a. Quanto tempo no total você gasta sentado durante um dia de semana?

horas minutos.

4b. Quanto tempo no total você gasta sentado durante em um dia de final de semana?

horas minutos. 